\title{
Klotho overexpression suppresses apoptosis by regulating the Hsp70/Akt/Bad pathway in H9c2(2-1) cells
}

\author{
JINPENG HU ${ }^{1,2^{*}}, \mathrm{BIN} \mathrm{SU}^{3 *}$, XUEWEN LI ${ }^{2}$, YUMING LI ${ }^{4,5}$ and JIHONG ZHAO ${ }^{2}$ \\ ${ }^{1}$ Graduate School of Tianjin Medical University, Tianjin 300070; ${ }^{2}$ Department of Geriatric Medicine, \\ Characteristic Medical Center of Chinese People's Armed Police Force; ${ }^{3}$ Ministry of Research, \\ Characteristic Medical Center of The Chinese People's Armed Police Force; ${ }^{4}$ Angiocardiopathy Institute of \\ Characteristic Medical Center of PAP, Tianjin 300162; ${ }^{5}$ TEDA International Cardiovascular Hospital, \\ Tianjin Economic-Technological Development Area, Tianjin 300457, P.R. China
}

Received April 11,2020; Accepted February 16, 2021

DOI: 10.3892/etm.2021.9917

\begin{abstract}
Early reperfusion is the most effective and important treatment for acute myocardial infarction. However, reperfusion therapy often leads to a certain degree of myocardial damage. The aim of the present study was to identify the role of klotho, and the molecular mechanism underlying its effects, in myocardial damage using a model of myocardial hypoxia injury. Hypoxia/reoxygenation $(\mathrm{H} / \mathrm{R})$ was used to mimic ischemia/reperfusion (I/R) injury in vitro. The expression and distribution of klotho in H9c2(2-1) cells was observed by fluorogenic scanning, and the apoptotic rate was determined by Annexin V-FITC/propidium iodide dual staining. Cell viability was determined by MTT assay, and caspase-3, cleaved caspase-3, Bcl-2, Bax, heat shock protein (Hsp) 70 and Akt levels were assessed by western blotting. A lactate dehydrogenase test was performed to determine the degree of $\mathrm{H} 9 \mathrm{c} 2(2-1)$ cell damage. The results revealed that klotho was primarily located in the cytoplasm of H9c2(2-1) cells. Klotho overexpression markedly suppressed H/R-induced H9c2(2-1) cell apoptosis. Furthermore, cell viability increased, and injury decreased in response to klotho. Klotho also suppressed the activation of caspase-3, upregulated $\mathrm{Bcl} 2$ and decreased Bax levels following $H / R$ injury, as well as alleviating $H / R$ injury by upregulating the expression of Hsp70 and increasing the levels of phosphorylated ( $\mathrm{p}-$ )Akt and Bad. In conclusion, these results indicate that klotho suppressed H/R-induced H9c2(2-1) cell apoptosis by regulating the levels of Hsp70, p-Akt and p-Bad, which suggest that klotho could be a novel agent for the treatment of coronary disease.
\end{abstract}

Correspondence to: Dr Yuming Li, Angiocardiopathy Institute of Characteristic Medical Center of PAP, 220 ChengLin Road, Tianjin 300162, P.R. China

E-mail: yumingli693@163.com

*Contributed equally

Key words: klotho, hypoxia/reoxygenation injury, apoptosis, H9c2(2-1) cells

\section{Introduction}

Globally, acute myocardial infarction (AMI) is associated with high mortality and morbidity rates $(1,2)$. Reperfusion therapy is the most effective way to treat this disease in a clinical setting (3). Though the therapeutic aim is to restore blood flow to the affected area, restoration of the blood supply often activates a cascade of cellular damage mechanisms, which may further aggravate myocardial cell pathology, namely myocardial ischemia/reperfusion injury (MIRI) (4). Cellular ischemic injury involves multiple injury mechanisms, such as abnormal calcium regulation in cells $(5,6)$, free radical production (7), mitochondrial damage (8) and the activation of apoptotic pathways (9). The cytotoxic cascade leads to the excessive production of reactive oxygen species (ROS), and is considered to be the primary factor responsible for myocardial contractile dysfunction, cell death and inflammation (10). These injuries may have serious consequences, such as heart failure or death. Therefore, there is an urgent requirement for further investigation of the pathogenesis of AMI, and to develop new therapeutic compounds and strategies.

The pathological mechanisms underlying ischemia-reperfusion injury (IRI) are complex, and include autophagy, cardiomyocyte apoptosis, cellular infiltration, calcium overload, oxidative stress, energy metabolism disorder and vascular endothelial dysfunction (11-13). There are multiple signaling pathways involved in I/R injury, such as the matrix metalloenzyme-associated, ATP-sensitive potassium channel, angiotensin II and Fas signaling pathways. Klotho is a protein that is involved in human aging and the length of the human lifespan $(14,15)$. A large number of previous studies have revealed that klotho is involved in the occurrence and development of various human diseases, including chronic kidney disease $(15,16)$, arteriosclerosis $(17)$, myocardial hypertrophy $(18,19)$, diabetes and obesity $(20-22)$, as well as various types of cancer $(23,24)$.

Klotho protein has been reported to exert anti- or pro-apoptotic functions in different diseases $(25,26)$. In an acute pancreatitis model, klotho was found to alleviate inflammation and apoptosis (27). Sugiura et al (28) found that klotho is involved in the pathophysiology of renal IRI, and 
that it alleviated apoptosis in a renal IRI model via heat shock protein (Hsp) 70. In an oxidative damage model, klotho was reported to attenuate oxidant-induced alveolar epithelial cell apoptosis and mitochondrial DNA damage (29). Moreover, klotho was also found to suppress ROS-induced apoptosis to improve cardiac function (30). In a stress-induced cardiac injury model, klotho inhibited cardiomyocyte apoptosis partly by suppressing the activation of the p38 and JNK pathways (31). Additionally, klotho inhibited the effects of dexamethasone via the NF- $\mathrm{B}$ signaling pathway in MC3T3-E1 osteoblasts (32). Moreover, in human umbilical vein endothelial cells, klotho suppressed apoptosis by reducing the activation of the PI3K/AKT pathway (33). Thus, klotho may be a potential therapeutic target for acute inflammatory disease.

The present study aimed to identify whether klotho exerts a protective effect on hypoxia/reoxygenation (H/R) injury in $\mathrm{H} 9 \mathrm{c} 2(2-1)$ cells, as well as the potential molecular mechanisms underlying this process. The results provide a useful reference for clarifying the molecular mechanisms of klotho during $\mathrm{H} / \mathrm{R}$ progression, and suggest klotho as a potential therapeutic target for acute MIRI.

\section{Materials and methods}

Regents and cell lines. The H9c2(2-1) rat heart myoblast cell line (ATCC ${ }^{\circledR}$ CRL-1446 ${ }^{\mathrm{TM}}$ ) was purchased from ATCC and cultured in Dulbecco's modified Eagle's medium (DMEM) supplemented with $10 \%$ fetal bovine serum. The pCMV3-C-HA negative control vector (C-terminal HA-tagged) and klotho cDNA ORF clone were purchased from Sino Biological, Inc. The klotho overexpression lentivirus and control plasmid lentivirus were constructed by and purchased from Kilton Biotechnology (Shanghai) Co., Ltd. Klotho shRNA (m) and control shRNA lentiviral particles (containing a scrambled shRNA sequence) were purchased from Santa Cruz Biotechnology, Inc.

$H / R$ injury model. H9c2(2-1) cells were precultured in serum-free DMEM overnight prior to H/R injury. Then, the cells were cultured and exposed to hypoxic conditions for $4 \mathrm{~h}$ using an AnaeroPack pouch (Mitsubishi Gas Chemical). The hypoxic conditions were $37^{\circ} \mathrm{C}, 2 \% \mathrm{O}_{2}$, and $5 \% \mathrm{CO}_{2}$. After $4 \mathrm{~h}$, reoxygenation was achieved by returning the cells to normal culture conditions, which involved incubation at $37^{\circ} \mathrm{C}$ in a humidified atmosphere containing $95 \%$ air and $5 \% \mathrm{CO}_{2}$ for $4 \mathrm{~h}$.

Transfection. H9c2(2-1) cells were plated into 6-well plate at the cell density of $4 \times 10^{5}$ cells/well $10 \mathrm{~h}$ prior to transfection. The transfection agent Lipofectamine ${ }^{\circledR} 2000$ was obtained from Invitrogen; Thermo Fisher Scientific, Inc. For transfection, $1 \mu \mathrm{g}$ pCMV3-C-HA negative control vector or $1 \mu \mathrm{g}$ klotho cDNA ORF clone was diluted in $100 \mu \mathrm{l}$ Opti-MEM (Thermo Fisher Scientific, Inc.); $4 \mu 1$ of Lipofectamine ${ }^{\circledR} 2000$ was diluted in $200 \mu \mathrm{l}$ of Opti-MEM. The mixtures were incubated at room temperature for $5 \mathrm{~min}$. Then, $100 \mu \mathrm{l}$ plasmid dilution liquid and $100 \mu \mathrm{l}$ diluted Lipofectamine ${ }^{\circledR} 2000$ liquid was mixed gently and kept at room temperature for $20 \mathrm{~min}$. The transfection mixture was added into each 6-well plate at room temperature. After $6 \mathrm{~h}$, the transfection medium was discarded and $2 \mathrm{ml}$ completed DMEM medium was added.
The transfected cells were cultured for $24 \mathrm{~h}$ and the target proteins such as caspase-3, Bcl2 and Bax were detected by western blotting after $\mathrm{H} / \mathrm{R}$ treatment.

Additionally, for shRNA lentiviral particles transduction, Klotho shRNA (m) and control shRNA lentiviral particles were used in order to knockdown klotho, H9c2(2-1) cells. Firstly, the cells were grown to $\sim 50 \%$ confluency and a mixture of complete DMEM medium with Polybrene ${ }^{\circledR}$ (cat. no. sc-134220; Santa Cruz Biotechnology, Inc.) at a final concentration of $5 \mu \mathrm{g} / \mathrm{ml}$ was prepared. $10 \mu \mathrm{l}$ klotho shRNA lentivirus or $10 \mu \mathrm{l}$ control shRNA lentivirus was added and cultured at $37^{\circ} \mathrm{C}$ for $24 \mathrm{~h}$. Subsequently, the culture medium was removed and the complete DMEM medium (without Polybrene ${ }^{\circledR}$ ) was replaced, and the cells were cultured for another $24 \mathrm{~h}$. The stable clones expressing the shRNA were selected and the levels of klotho, p-Akt and total Akt were detected by western blotting. Percentages of apoptotic H9c2(2-1) cells transfected with klotho or control vector were subsequently investigated.

Flow cytometric analysis. H9c2(2-1) cells were infected with klotho lentivirus or control plasmid lentivirus for $24 \mathrm{~h}$ prior to $\mathrm{H} / \mathrm{R}$ treatment. Apoptosis was assessed by Annexin V-FITC/propidium iodide (PI) staining according to the kit protocols (Santa Cruz Biotechnology, Inc.). For flow cytometric analysis, the cells were digested with $0.25 \%$ trypsin for $1 \mathrm{~min}$ and washed twice with precooled phosphate-buffered saline (PBS). The cells were then resuspended in $500 \mu \mathrm{l}$ binding buffer with Annexin V-FITC $(0.1 \mu \mathrm{g} / \mu \mathrm{l})$ and PI $(0.05 \mu \mathrm{g} / \mu \mathrm{l})$ in the dark for $15 \mathrm{~min}$ on ice. From each sample, $1 \times 10^{4}$ cells were collected for detection. The samples were then run through a flow cytometer (BD LSRFortessa X-20; BD Biosciences) and the data was analyzed using FlowJo v10 software (FlowJo LLC).

MTT assay. Cell viability was determined with an MTT assay. Briefly, H9c2(2-1) cells were transfected with klotho or negative control lentivirus for $24 \mathrm{~h}$. Then, $3 \times 10^{4}$ cells/well were plated into 96 -well plates for $6 \mathrm{~h}$ and treated by H/R injury as aforementioned. Then, $10 \mu \mathrm{l}$ MTT reagent was added to each well and the plate was cultured for $4 \mathrm{~h}$ at $37^{\circ} \mathrm{C}$. The absorbance was measured at $570 \mathrm{~nm}$ with a microplate reader, and viability was calculated as follows: Cell viability $=\left(\mathrm{OD}_{570 \mathrm{~nm}}\right.$ of treatment group-OD $\mathrm{D}_{570 \mathrm{~nm}}$ of blank wells $) /\left(\mathrm{OD}_{570 \mathrm{~nm}}\right.$ of untreated group- $\mathrm{OD}_{570 \mathrm{~nm}}$ of blank wells) x100\%.

Lactate dehydrogenase $(L D H)$ assay. Cellular injury was evaluated using the LDH test. LDH is a stable and abundant cytoplasmic enzyme that is unable to pass through the cell membrane. When a cell is damaged or dead, LDH is quickly released into the cell culture medium. Thus, LDH activity in the supernatant is proportional to the number of dead cells. Briefly, H9c2(2-1) cells were infected with klotho lentivirus or control plasmid lentivirus for $24 \mathrm{~h}$ prior to H/R treatment. Cell supernatants were obtained from each group and LDH activity was determined using an $\mathrm{LDH}$ release assay according to the manufacturer's protocol (Nanjing KeyGen Biotech Co., Ltd.).

Scanning confocal microscopy. H9c2(2-1) cells were infected with klotho lentivirus or control plasmid lentivirus for $24 \mathrm{~h}$ prior to $H / R$ treatment. Then, the cells were washed twice with PBS and fixed with $100 \%$ methanol for $5 \mathrm{~min}$ at room temperature. 


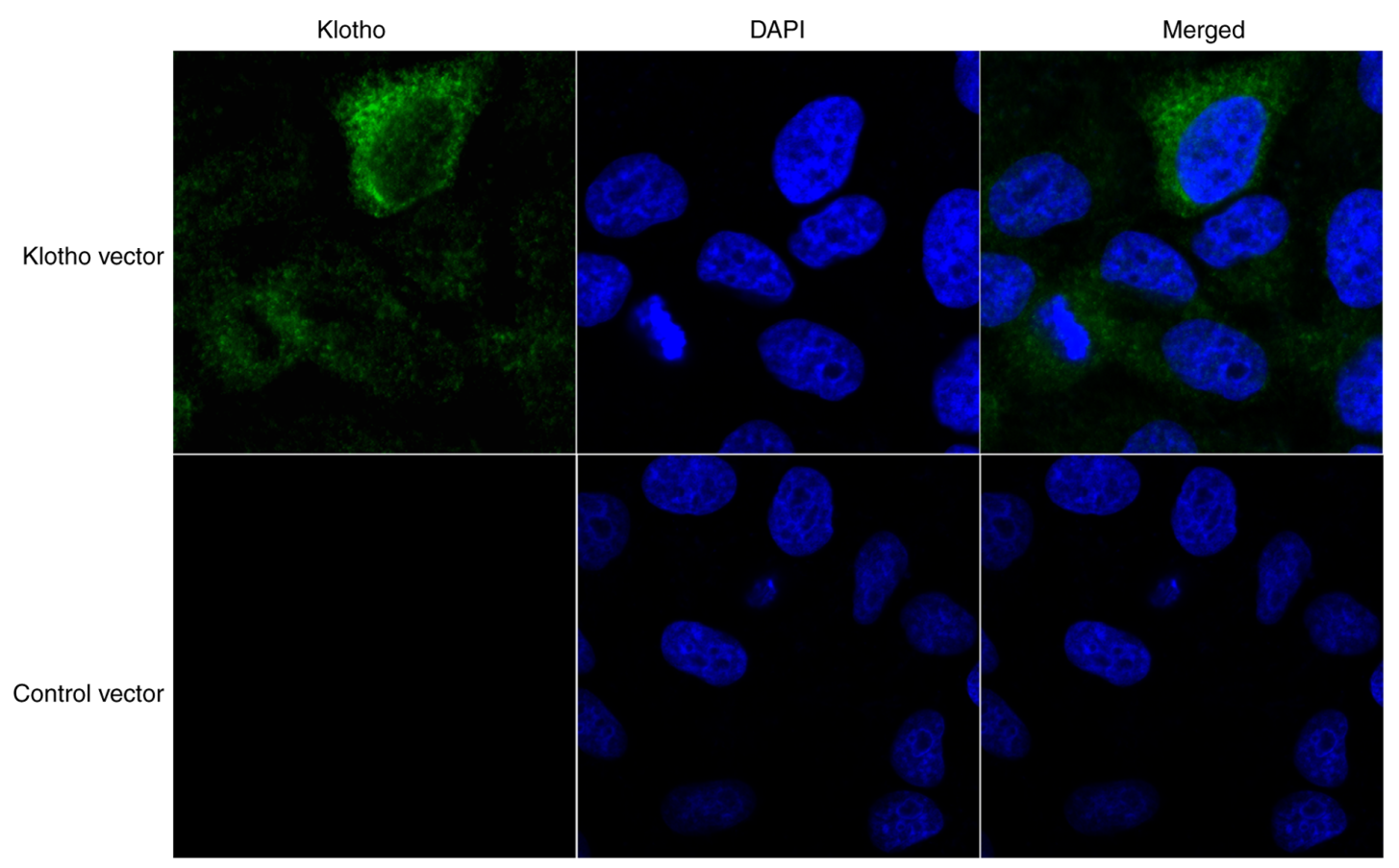

Figure 1. Klotho is primarily located in the cytoplasm of cardiomyocytes. H9c2(2-1) cardiomyocytes were transfected with a klotho expression vector or its negative pCMV3 control vector for $24 \mathrm{~h}$. Confocal microscopy images showed that klotho was located in the cytoplasm of H9c2(2-1) cells.

Next, the cells were permeabilized with $0.1 \%$ Triton $\mathrm{X}-100$ for $5 \mathrm{~min}$ and treated with $2 \% \mathrm{BSA}$ in $0.1 \%$ PBS-Tween for $1 \mathrm{~h}$ to block non-specific protein-protein interactions at room temperature. Primary Hsp70 antibody was added, and the cells were incubated overnight at $4^{\circ} \mathrm{C}$. The Anti-Hsp70 antibody [EPR16892 (Alexa Fluor ${ }^{\circledR}$ 647; cat. no. ab204691)] was purchased from Abcam. The cell nuclei were stained with DAPI at a concentration of $1.43 \mu \mathrm{M}$, and were observed by fluorescence confocal microscopy at x200 magnification. In an alternative assay, H9c2(2-1) cells were infected, permeabilized and blocked as aforementioned, and then stained with Hoechst 33258 (Qcbio Science \& Technologies Co. Ltd.) to assess the effect of klotho overexpression on the morphology of H/R-induced apoptotic cells.

Western blot analysis. The levels of caspase-3, Bax, Bcl-2, klotho, Hsp70, phosphorylated (p-)Akt, Akt, p-Bad and Bad were detected by western blotting. Cell lysis buffer was purchased from Beyotime Institute of Biotechnology (cat. no. P0013). Briefly, cell lysates were prepared and total protein concentration was determined using a BCA Protein Assay kit (Thermo Fisher Scientific, Inc.). A $20-\mu$ g sample of total protein was loaded into each lane and separated by $10 \%$ SDS-PAGE. The proteins were then transferred onto PVDF membranes at $300 \mathrm{~mA}$ for $2 \mathrm{~h}$. The membranes were blocked with $5 \%$ non-fat milk at room temperature for $40 \mathrm{~min}$, and then incubated with primary antibodies at a ratio of 1:2,000 overnight at $4^{\circ} \mathrm{C}$. The following primary antibodies were used: Anti-caspase-3 (cat. no. 3138-100; BioVision, Inc.), anti-Bcl-2 (cat. no. AP1303a-ev; Abgent, Inc.), anti-Bax (cat. no. AP1302a-ev; Abgent, Inc.), recombinant anti-Hsp70 (EPR16892; cat. no. ab181606; Abcam); anti-Klotho (EPR6856; cat. no. ab181373; Santa Cruz Biotechnology, Inc.); anti-p-Akt (B-5; cat. no. sc-271966; Santa Cruz Biotechnology, Inc.), anti-Akt (EPR16798; cat. no. ab179463; Abcam), anti-Bad [phospho S112 (EPR1891(2)); cat. no. ab129192; Abcam]; anti-Bad (cat. no. ab90435; Abcam) and $\beta$-actin (cat. no. AM1021B; Abgent, Inc.). The membrane was then incubated with horseradish peroxidase-conjugated goat anti-rabbit secondary antibody (1:10,000, cat. no. sc-2004; Santa Cruz Biotechnology, Inc.) for $1 \mathrm{~h}$ at room temperature. The protein bands were detected in a dark room using an ECL detection kit (Cytiva).

Statistical analysis. All results were analyzed with SPSS 20.0 software (IBM Corp) using one-way analysis of variance followed by Tukey's post hoc test. The independent samples were analyzed using an unpaired t-test, including the comparison between klotho protein expression in H/R-injured cells and control H9c2(2-1) cells. $\mathrm{P}<0.05$ was considered to indicate a statistically significant difference.

\section{Results}

Klotho is primarily located in the cytoplasm of H9c2(2-1) cells. In order to determine the role of klotho in the H/R cardiomyocyte model, H9c2(2-1) cells were transfected the klotho cDNA ORF clone and its negative control pCMV3-C-GFPSpark vector (with C-terminal GFPSpark-tag). After $24 \mathrm{~h}$, the distribution of klotho in H9c2(2-1) cells was determined by confocal microscopy. As shown in Fig. 1, klotho was primarily distributed in the cytoplasm of H9c2(2-1) cells.

Overexpression of klotho markedly inhibits the $H / R$-induced apoptosis of H9c2(2-1) cells. Klotho levels were first detected in H/R-treated H9c2(2-1) cells by western blotting. As shown in Fig. 2A, klotho protein expression was reduced by $H / R$ injury. Additionally, to further confirm the role of klotho in apoptosis, Hoechst 33258 was used to stain the nuclei and to 
A

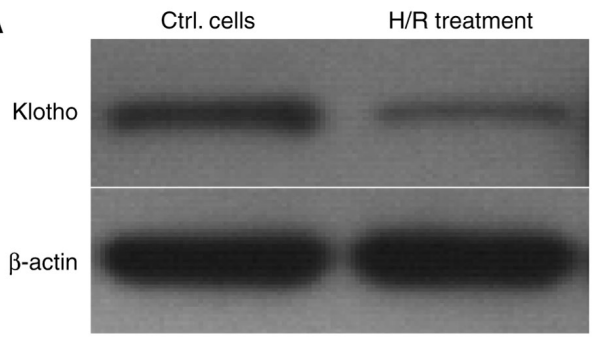

B

H/R injury
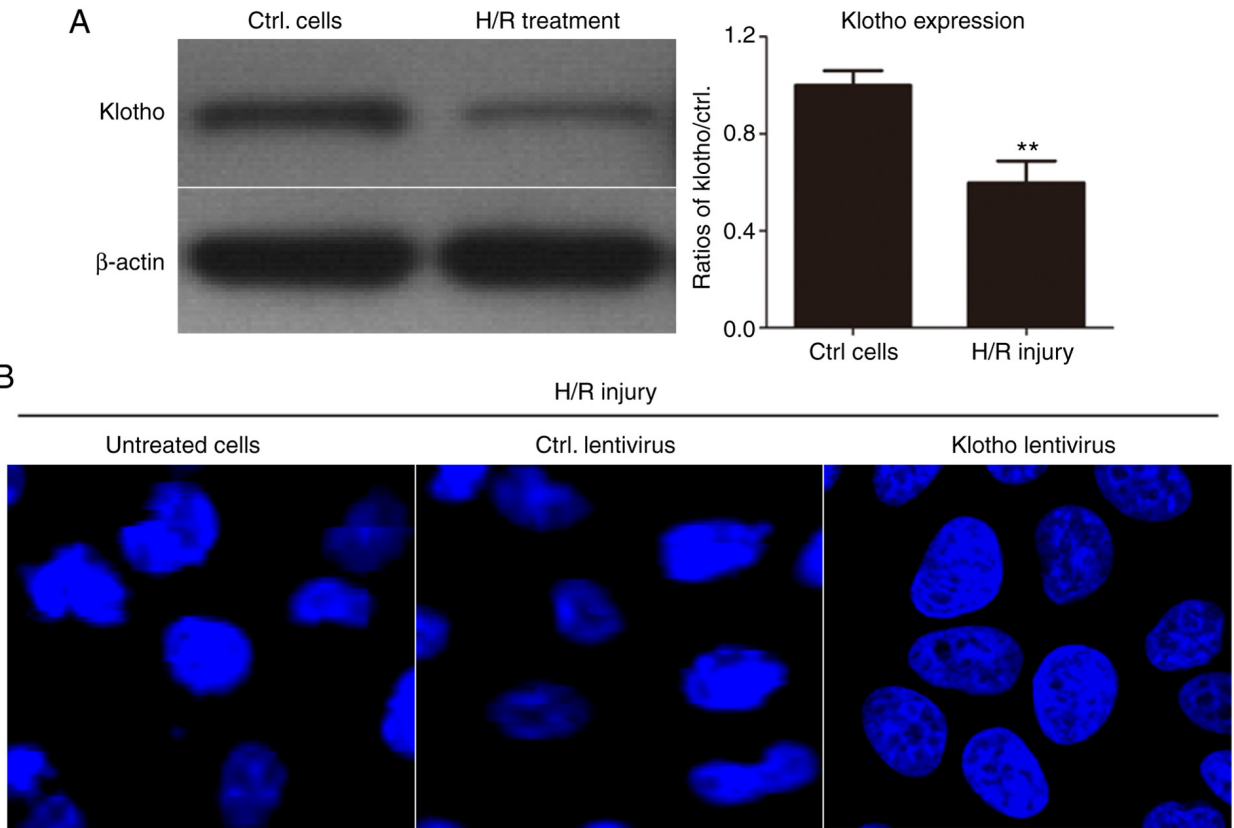

C
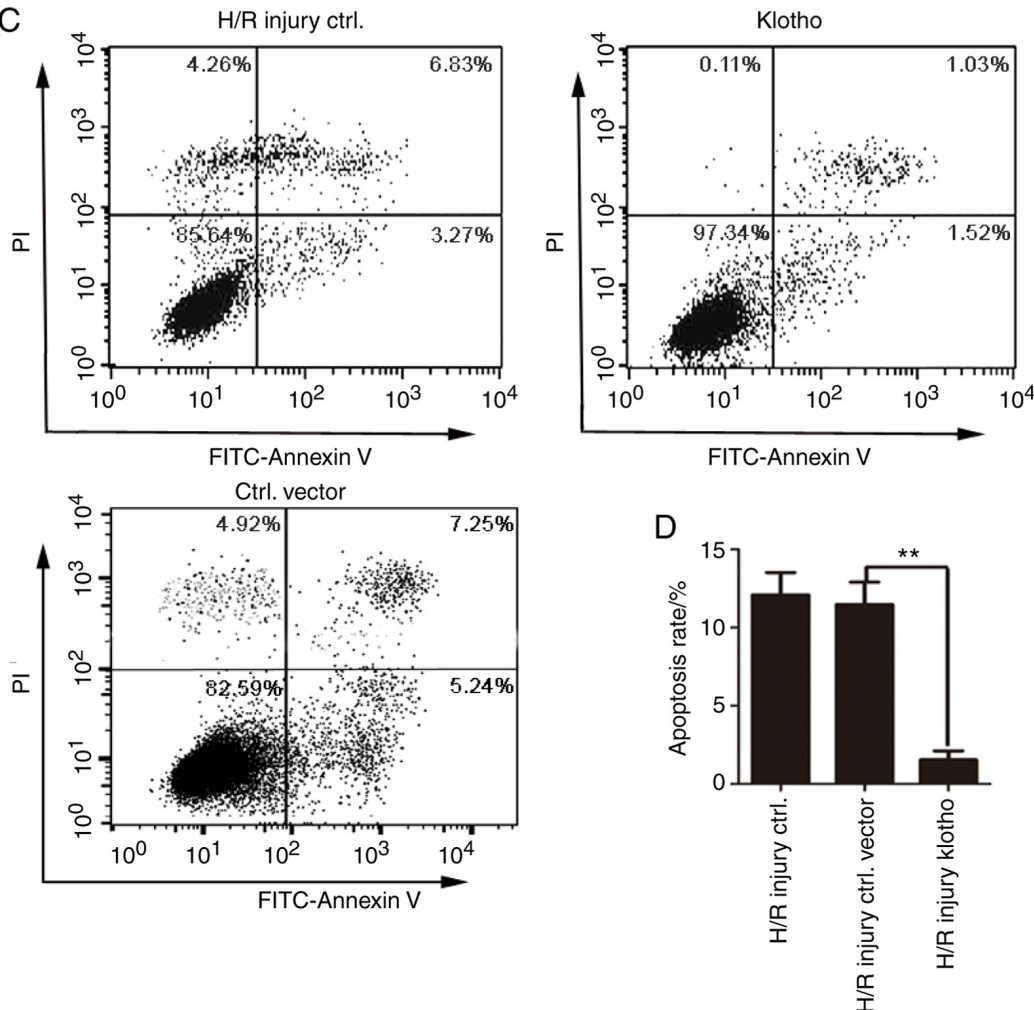

Figure 2. Klotho overexpression markedly inhibits H/R-induced apoptosis in H9c2(2-1) cells. (A) Klotho protein expression induced by H/R injury in H9c2(2-1) cells. ${ }^{* *} \mathrm{P}<0.01$ compared with untreated cells. (B) Confocal microscopy was used to detect the effects of klotho overexpression on the morphology of H/R-induced apoptotic H9c2(2-1) cells using Hoechst 33258. (C) Cardiomyocyte apoptosis was determined by Annexin V and PI staining. Percentages of Annexin V-positive cells of the total number of cells were counted. (D) Percentages of apoptotic H9c2(2-1) cells transfected with klotho or control vector are shown in the histogram. ${ }^{* *} \mathrm{P}<0.01$, compared with control vector-transfected H9C2 cells. H/R, hypoxia/reoxygenation; PI, propidium iodide; Ctrl, control.

detect the effects of klotho overexpression on the morphology of H/R-induced H9c2(2-1) cells. The results revealed that the nuclear membrane of some cells shrank, the nuclear chromatin appeared dense with enhanced fluorescence staining, and nuclear fragmentation and apoptotic body formation were apparent in a number of cells. Compared with those of the control cells, the nuclei of the klotho lentivirus-infected $\mathrm{H} 9 \mathrm{c} 2(2-1)$ cells were smooth, intact and uniform in density, and the chromatin in the nucleus was evenly stained. These results revealed that klotho overexpression alleviated apoptosis induced by H/R injury (Fig. 2B).

To determine the role of klotho in H/R-induced apoptosis, H9c2(2-1) cells were infected with klotho lentivirus or the control vector lentivirus for $24 \mathrm{~h}$ followed by H/R treatment. The apoptotic rate in each group was determined by Annexin V-FITC/PI dual staining. As shown in Fig. 2C, under 
A

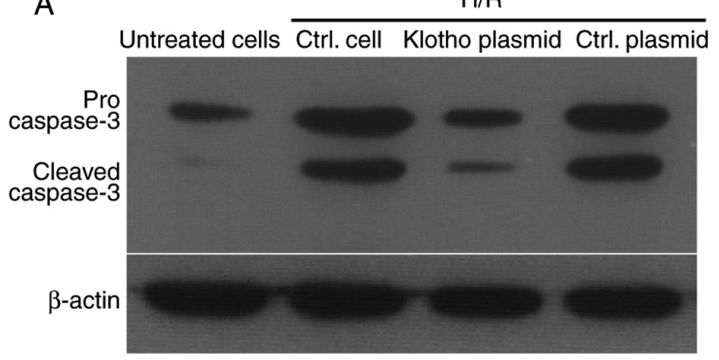

B

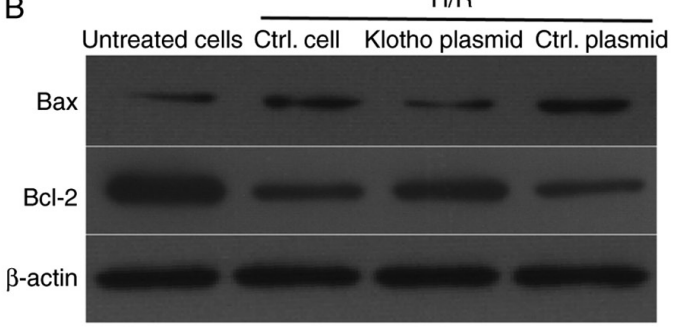

Figure 3. Klotho suppresses the activation of caspase-3, upregulates Bcl2, and decreases the level of Bax after H/R treatment. H9c2(2-1) cells were transfected with klotho or control plasmids for $24 \mathrm{~h}$, and were then treated with H/R. (A) Levels of pro-caspase-3 and cleaved caspase-3, and (B) Bax and Bcl-2 were detected by western blotting. $\beta$-actin was used as the internal reference gene. H/R, hypoxia/reoxygenation; Ctrl, control.
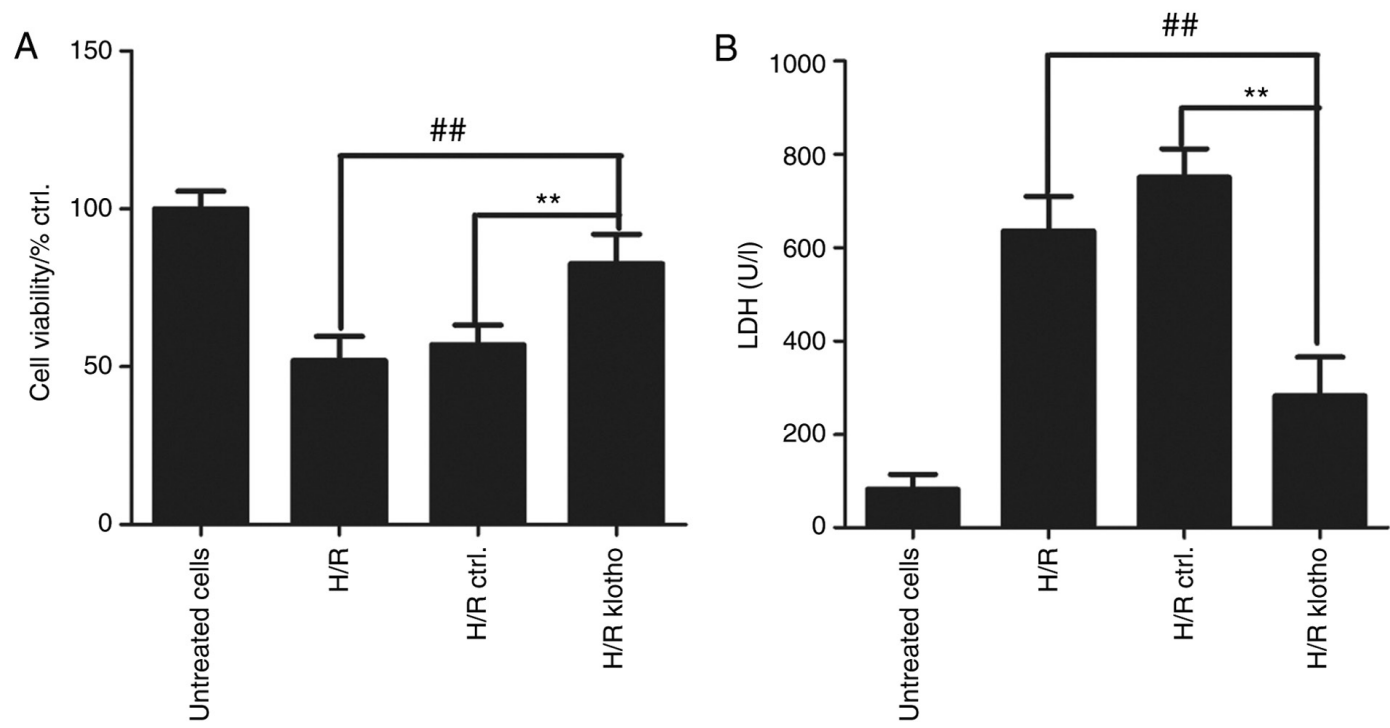

Figure 4. Klotho retains viability and decreases injury in H9c2(2-1) cardiomyocyte cells. H9c2(2-1) cells were transfected with a klotho plasmid or control vector for $24 \mathrm{~h}$ prior to $\mathrm{H} / \mathrm{R}$ treatment. (A) MTT was used to assess cellular viability in each group. ${ }^{* *} \mathrm{P}<0.01$ and ${ }^{\# \#} \mathrm{P}<0.01$. (B) LDH assay was performed to determine the degree of cardiomyocyte damage. LDH levels were tested using cultured medium ${ }^{* *} \mathrm{P}<0.01$ and ${ }^{\# \#} \mathrm{P}<0.01$. H/R, hypoxia/reoxygenation; Ctrl, control; LDH, lactate dehydrogenase.

$\mathrm{H} / \mathrm{R}$ conditions, the apoptotic rate was significantly higher in control vector lentivirus-infected cells. However, infection with klotho plasmid lentivirus significantly inhibited the apoptosis with $H / R$ injury. These results revealed that $H / R$ treatment markedly increased the number of apoptotic cells, while klotho overexpression inhibited the H/R-induced apoptosis of H9c2(2-1) cells.

Klotho suppresses the activation of caspase-3, upregulates Bcl2 and downregulates Bax expression following H/R treatment. In order to investigate the inhibitory effects of klotho on $\mathrm{H} 9 \mathrm{c} 2(2-1)$ cell apoptosis after H/R treatment, the levels of proand cleaved caspase- 3 were detected by western blotting. As shown in Fig. 3A, H/R treatment increased the levels of proand cleaved caspase-2 in H9c2(2-1) cells, while infection with the klotho lentivirus inhibited the increase in these proteins, compared with those in the control plasmid-infected group. Moreover, the ratio of Bcl-2 to Bax is a key factor in reflecting apoptosis induced by various stimuli. Thus, the levels of Bcl-2 and Bax were also detected in H/R-treated H9c2(2-1) cells after infection with klotho lentivirus or control vector lentivirus. As shown in Fig. 3B, klotho induced a higher level of Bcl-2 and decreased the level of Bax in H9c2(2-1) cells compared with the control vector lentivirus after $H / R$ injury. These results demonstrate that $\mathrm{H} / \mathrm{R}$ treatment promoted the activation of pro-caspase-3, and that klotho overexpression inhibited H/R injury-induced apoptosis by inhibiting caspase-3 activation and increasing the ratio of $\mathrm{Bcl}-2$ to Bax. Taken together, these data reveal that klotho has an anti-apoptotic effect on $\mathrm{H} / \mathrm{R}$-induced apoptosis in H9c2(2-1) cells.

Klotho retains cell viability and decreases $H 9 c 2(2-1)$ cell injury. MTT and LDH assays were used to evaluate the effects of klotho on myocardial cell viability and damage after $\mathrm{H} / \mathrm{R}$ treatment. Cardiomyocytes were treated with $\mathrm{H} / \mathrm{R}$ for $4 \mathrm{~h}$, and the cells were then transfected with klotho or control plasmid for $24 \mathrm{~h}$. As shown in Fig. 4A, cell viability decreased after $\mathrm{H} / \mathrm{R}$ treatment for $4 \mathrm{~h}$, and klotho retained H9c2(2-1) cell viability following H/R treatment ( $\mathrm{P}<0.01$ compared with the control group). The level of LDH can represent the degree of cardiomyocyte damage, and cardiomyocyte injury results in increased LDH levels. As shown in Fig. 4B, LDH levels were significantly higher in H/R-treated cells than in normal cells, suggesting that $\mathrm{H} / \mathrm{R}$ results in cellular injury. The level of LDH in the cardiomyocyte culture medium 


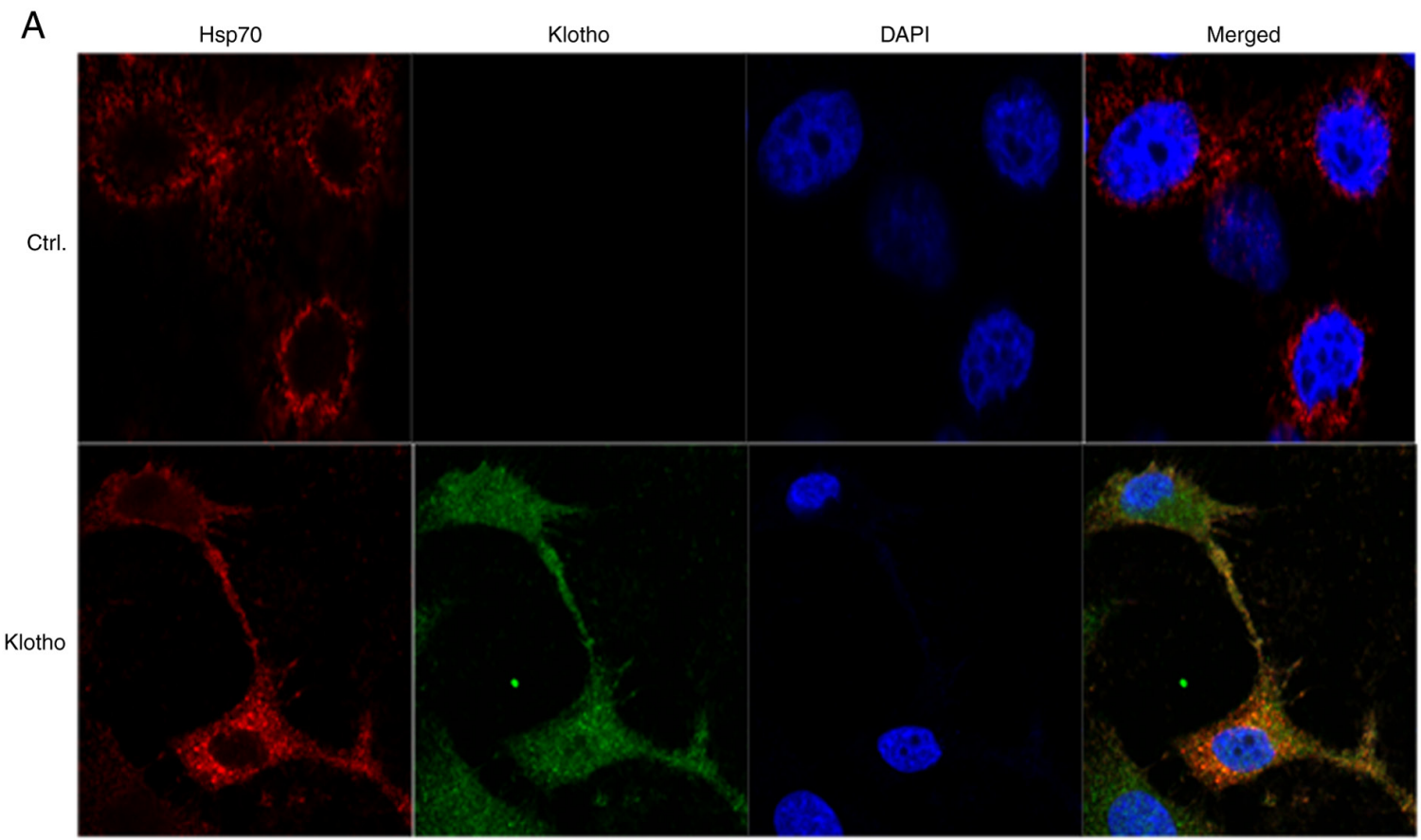

B
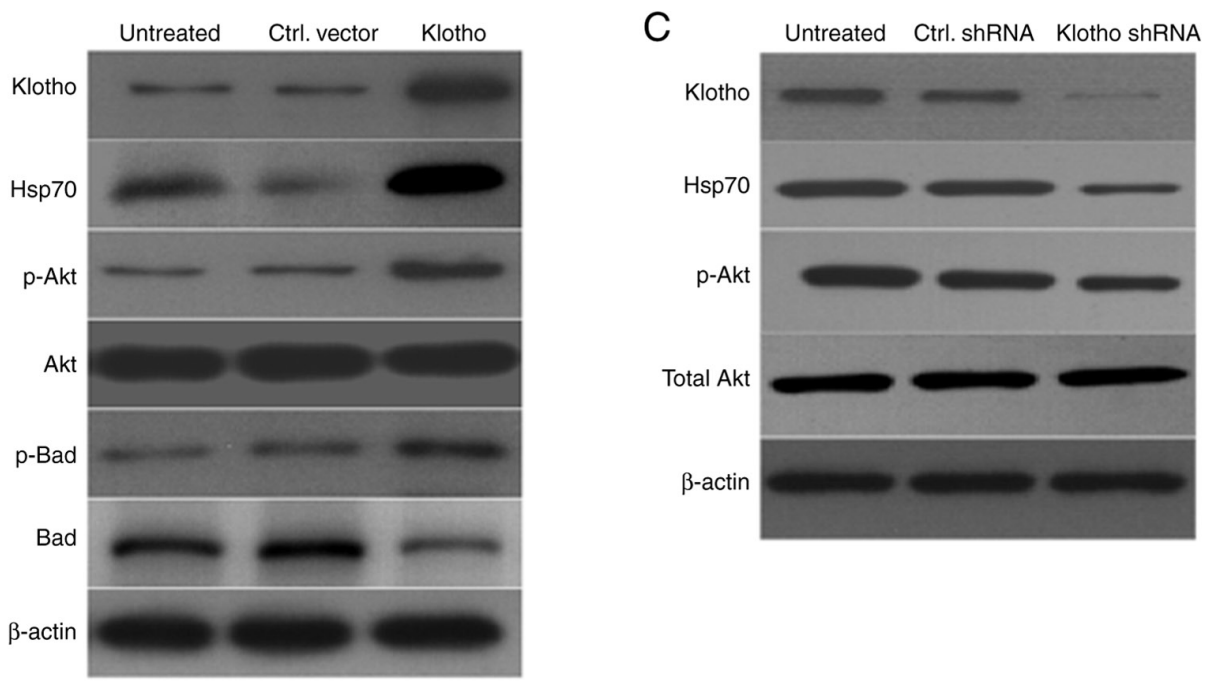

Figure 5. Klotho mitigates H/R injury by regulating the Hsp70/Akt/Bad pathway. (A) H9c2(2-1) cells were transfected with klotho plasmid for $24 \mathrm{~h}$ prior to $\mathrm{H} / \mathrm{R}$ injury. Expression levels of klotho and Hsp70 were detected by immunofluorescence microscopy (magnification, x200). DAPI was used to stain the nuclei. (B) Expression of klotho, p-Akt, p-Bad, total Akt and total Bad was detected by western blotting. $\beta$-actin was used as the internal reference gene. (C) H9c2(2-1) cells were infected with klotho shRNA lentivirus or control shRNA lentivirus for $48 \mathrm{~h}$. The levels of klotho, p-Akt and total Akt were detected by western blotting. H/R, hypoxia/reoxygenation; Ctrl, control; Hsp70, heat shock protein 70; p-, phosphorylated; sh, short hairpin.

was significantly lower in the klotho-transfected group than in the control group $(\mathrm{P}<0.01)$. These results demonstrate that klotho reduces myocardial cell damage.

Overexpression of klotho in H/R-treated H9c2(2-1) cells increases the levels of Hsp70, p-Akt and p-Bad. It has been reported that klotho mitigates apoptosis in cells involved in ischemic acute kidney injury by regulating the level of Hsp70 (28). The present study investigated whether Hsp70 was involved in IRI in klotho-overexpressing H9c2(2-1) cells. Firstly, cells were infected with klotho lentivirus for $24 \mathrm{~h}$ prior to H/R treatment, and Hsp70 expression was detected by confocal laser scanning microscopy and western blotting. As shown in Fig. 5A, Hsp70 and klotho were colocalized in the cytoplasm of H/R-treated cells. Moreover, western blotting revealed that klotho overexpression in $\mathrm{H} 9 \mathrm{c} 2(2-1)$ cells with $\mathrm{H} / \mathrm{R}$ injury was accompanied by increased levels of Hsp70, p-Akt and p-Bad; the level of total Akt was not markedly altered, and total Bad was markedly decreased in klotho lentivirus-infected cells (Fig. 5B). Conversely, klotho-knockdown with $\mathrm{H} / \mathrm{R}$ injury resulted in decreased expression of Hsp70 and p-Akt, though total Akt levels were unchanged in klotho shRNA lentivirus-infected cells (Fig. 5C). These results show that klotho overexpression alleviated H/R injury in H9c2(2-1) cells by upregulating the levels of Hsp70, p-Akt and p-Bad.

\section{Discussion}

AMI is a disease with high global incidence and mortality rates (34). MIRI, which refers to the interruption of blood 
supply to the myocardium during pathological injury, is the most common cause of AMI (35). After the blood supply is restored, the original ischemic myocardial injury does not improve, but instead shows more serious damage than that prior to blood supply recovery. In the present study, a H/R injury model was constructed using H9c2(2-1) cells to mimic acute MIRI. The protective role of klotho on myocardial injury was subsequently identified, and klotho was found to suppress apoptosis by upregulating the levels of Hsp70, p-Akt and $\mathrm{p}$-Bad.

$\mathrm{H} / \mathrm{R}$ injury significantly increased the apoptotic rate of H9c2(2-1) cells and also resulted in decreased klotho expression. Apoptosis was flow cytometrically evaluated by Annexin V-FITC/PI dual staining, and the results showed that the apoptotic rate of $\mathrm{H} 9 \mathrm{c} 2(2-1)$ cells was markedly increased in the $\mathrm{H} / \mathrm{R}$ injury group compared with that of the control group. Furthermore, klotho overexpression prior to $\mathrm{H} / \mathrm{R}$ treatment significantly inhibited H/R-induced apoptosis. The effect of klotho on H9c2(2-1) cell viability was subsequently assessed using MTT. The results indicated that H/R treatment reduced the survival rate of cardiomyocytes and that klotho overexpression significantly inhibited cell injury and death. Additionally, when myocardial cells are damaged, LDH level increases, thus LDH level represents the degree of myocardial cell damage. An LDH assay was performed to assess $\mathrm{H} 9 \mathrm{c} 2(2-1)$ cell injury following $\mathrm{H} / \mathrm{R}$; the results showed that $\mathrm{H} / \mathrm{R}$ treatment increased $\mathrm{LDH}$ release, suggesting that $\mathrm{H} / \mathrm{R}$ results in cardiomyocyte injury. However, the LDH level in the cardiomyocyte culture medium of the klotho overexpression group was significantly lower than that in the control group $(\mathrm{P}<0.01)$, suggesting that klotho overexpression inhibited $\mathrm{H} 9 \mathrm{c} 2(2-1)$ cell damage.

Next, the molecular mechanism by which klotho inhibits apoptosis was investigated by western blotting. The results showed that klotho overexpression inhibited the activation of caspase-3, increased the $\mathrm{Bcl}-2 / \mathrm{Bax}$ ratio, as well as the level of p-Bad in H9c2(2-1) cells, compared with those in control vector lentivirus-infected cells. Furthermore, colocalization of Hsp70 and klotho was noted in the cytoplasm of H/R-treated cells via confocal laser scanning microscopy and western blotting. These results suggest that klotho and Hsp70 were co-localized in the cytoplasm and inhibited the apoptosis of H/R-treated cells via hsp70. This was consistent with the findings of Sugiura et al (28), which showed that in an ischemic acute kidney injury model, klotho inhibited apoptosis via Hsp70. Previously, Hsp90 was found to exert a profound ischemic postconditioning cardioprotective effect and to alleviate I/R-induced myocardial injury and apoptosis in vivo (36). Notably, the western blotting results of the present study showed that klotho overexpression in $\mathrm{H} 9 \mathrm{c} 2(2-1)$ cells prior to $H / R$ treatment upregulated the levels of p-Akt and p-Bad. The total Akt level was not notably altered, and the total Bad level was markedly decreased in klotho-overexpressing cells. Together, these data suggest that klotho inhibited apoptosis by interacting with Hsp70 and decreasing the levels of Bad in H9c2(2-1) cells.

As mature cardiac myocytes are unable to proliferate, their survival is important in maintaining cardiac health and function. The proliferative abilities and apoptosis of cardiomyocytes after MIRI have been widely studied. Although the antiaging protein klotho reportedly possesses a protective role in cardiac diseases, the precise mechanisms underlying this effect remain unknown. A previous study indicated that klotho inhibited angiotensin II-induced cardiomyocyte hypertrophy by suppressing the angiotensin II type I receptor/ $\beta$-catenin pathway (1). Another group found that klotho suppressed cardiomyocyte apoptosis in mice with stress-induced cardiac injury by downregulating endoplasmic reticulum stress (2). In the present study, klotho and HSP70 were found to co-localize in the cell cytoplasm, and klotho overexpression was accompanied by HSP70 upregulation. Thus, how klotho exerts its protective role in the $\mathrm{H} / \mathrm{R}$ cardiomyocytes by regulating the expression of Hsp70 was further investigated. However, as the present study was comprised solely of in vitro experiments, in vivo functions should be investigated in the future.

In the present study, the role of klotho in H/R injury-induced proliferation and apoptosis after MIRI was investigated, and the associated molecular mechanism was further clarified. Collectively, the results indicate that klotho reduces apoptosis by upregulating Hsp70 and p-Akt after IRI, suggesting that klotho may serve as a potential therapeutic target for $\mathrm{I} / \mathrm{R}$ injury and repair.

\section{Acknowledgements}

Not applicable.

\section{Funding}

The present study was supported by the National Key R\&D Plan (grant no.2017YFC1307602), the Tianjin Science and Technology Commission Support Plan (grant no. 15ZXLCSY00040).

\section{Availability of data and materials}

The datasets used and/or analyzed during the current study are available from the corresponding author on reasonable request.

\section{Author's contributions}

$\mathrm{JH}$ checked the references and performed several western blotting assays. BS performed the flow cytometry and several western blotting experiments. XL and JZ performed the MTT assay, acquired the data and prepared the manuscript. YL designed the experiments and wrote the manuscript. All authors read and approved the final manuscript. JH and YL were responsible for the authenticity of the raw data.

\section{Ethics approval and consent to participate}

Not applicable.

\section{Patient consent for publication}

Not applicable.

\section{Competing interests}

The authors declare that they have no competing interests 


\section{References}

1. Alabas OA, Jernberg T, Pujades-Rodriguez M, Rutherford MJ, West RM, Hall M, Timmis A, Lindahl B, Fox KA, Hemingway $\mathrm{H}$, et al: Statistics on mortality following acute myocardial infarction in 842897 Europeans. Cardiovasc Res 116: 149-157, 2020.

2. Ioacara S, Popescu AC, Tenenbaum J, Dimulescu DR, Popescu MR, Sirbu A and Fica S: Acute Myocardial Infarction Mortality Rates and Trends in Romania between 1994 and 2017 Int J Environ Res Public Health 17: 285, 2019.

3. Neri M, Riezzo I, Pascale N, Pomara C and Turillazzi E: Ischemia/ reperfusion injury following acute myocardial infarction: A critical issue for clinicians and forensic pathologists. Mediators Inflamm 2017: 7018393, 2017.

4. Wang M and Wan J: Soluble regulators of Interleukin-1 signaling: Novel biomarkers for early acute myocardial infarction diagnosis and to predict ischemia/reperfusion injury? Int J Cardiol 274: $357,2019$.

5. Feng M, Wang Q, Wang $\mathrm{H}$ and Guan W: Tumor necrosis factor-alpha preconditioning attenuates liver ischemia/reperfusion injury through preserving sarco/endoplasmic reticulum calcium-ATPase function. J Surg Res 184: 1109-1113, 2013.

6. Li X, Dai Y, Yan S, Shi Y, Han B, Li J, Cha L and Mu J: Down-regulation of 1 ncRNA KCNQ1OT1 protects against myocardial ischemia/reperfusion injury following acute myocardial infarction. Biochem Biophys Res Commun 491: 1026-1033, 2017.

7. Tu CC, Wan BY and Zeng Y: STIM 2 knockdown protects against ischemia/reperfusion injury through reducing mitochondrial calcium overload and preserving mitochondrial function. Life Sci 247: 116560, 2020.

8. Yue R, Xia X, Jiang J, Yang D, Han Y, Chen X, Cai Y, Li L, Wang WE and Zeng C: Mitochondrial DNA oxidative damage contributes to cardiomyocyte ischemia/reperfusion-injury in rats: Cardioprotective role of lycopene. J Cell Physiol 230: 2128-2141, 2015

9. Wang Z, Sun R, Wang G, Chen Z, Li Y, Zhao Y, Liu D, Zhao H, Zhang F, Yao J, et al: SIRT3-mediated deacetylation of PRDX3 alleviates mitochondrial oxidative damage and apoptosis induced by intestinal ischemia/reperfusion injury. Redox Biol 28: 101343, 2020.

10. Wang X, He F, Liao Y, Song X, Zhang M, Qu L, Luo T, Zhou S, Ling Y, Guo J, et al: Baicalin pretreatment protects against myocardial ischemia/reperfusion injury by inhibiting mitochondrial damage-mediated apoptosis. Int J Cardiol 168 : 4343-4345, 2013

11. Yang Y, Duan W, Jin Z, Yi W, Yan J, Zhang S, Wang N, Liang Z, Li Y, Chen W, et al: JAK2/STAT3 activation by melatonin attenuates the mitochondrial oxidative damage induced by myocardial ischemia/reperfusion injury. J Pineal Res 55: 275-286, 2013

12. Xie Y, Jiang D, Xiao J,Fu C, Zhang Z, YeZ and Zhang X: Ischemic preconditioning attenuates ischemia/reperfusion-induced kidney injury by activating autophagy via the SGK1 signaling pathway. Cell Death Dis 9: 338, 2018.

13. Zhou LY, Zhai M, Huang Y, Xu S, An T, Wang YH, Zhang RC, Liu CY, Dong YH, Wang M, et al: The circular RNA ACR attenuates myocardial ischemia/reperfusion injury by suppressing autophagy via modulation of the Pink1/ FAM65B pathway. Cel Death Differ 26: 1299-1315, 2019.

14. Di Bona D, Accardi G, Virruso C, Candore G and Caruso C: Association of Klotho polymorphisms with healthy aging: A systematic review and meta-analysis. Rejuvenation Res 17: 212-216, 2014

15. Wang Q, Su W, Shen Z and Wang R: Correlation between soluble alpha-Klotho and renal function in patients with chronic kidney disease: A Review and meta-analysis. Biomed Res Int 2018 9481475, 2018.

16. Mosa O, Skitek M and Jerin A: Validity of Klotho, CYR61 and YKL-40 as ideal predictive biomarkers for acute kidney injury: Review study. Sao Paulo Med J 135: 57-65, 2017.

17. Kuwahara N, Sasaki S, Kobara M, Nakata T, Tatsumi T, Irie H, Narumiya H, Hatta T, Takeda K, Matsubara H, et al: HMG-CoA reductase inhibition improves anti-aging klotho protein expression and arteriosclerosis in rats with chronic inhibition of nitric oxide synthesis. Int J Cardiol 123: 84-90, 2008.

18. Yang K, Wang C, Nie L, Zhao X, Gu J, Guan X, Wang S, Xiao T, Xu X, He T, et al: Klotho protects against indoxyl sulphate-induced myocardial hypertrophy. J Am Soc Nephrol 26 2434-2446, 2015.
19. Ramez M, Rajabi H, Ramezani F, Naderi N, Darbandi-Azar A and Nasirinezhad F: The greater effect of high-intensity interval training versus moderate-intensity continuous training on cardioprotection against ischemia-reperfusion injury through Klotho levels and attenuate of myocardial TRPC6 expression. BMC Cardiovasc Disord 19: 118, 2019.

20. Takenaka T, Kobori H, Miyazaki T, Suzuki H, Nishiyama A, Ishii N, Yamashita M and Hayashi M: Klotho protein supplementation reduces blood pressure and renal hypertrophy in $\mathrm{db} / \mathrm{db}$ mice, a model of type 2 diabetes. Acta Physiol (Oxf) 225: e13190, 2019.

21. Donate-Correa J, Martín-Núñez E, Delgado NP, de Fuentes MM, Arduan AO, Mora-Fernández $\mathrm{C}$ and Navarro González JF: Implications of Fibroblast growth factor/Klotho system in glucose metabolism and diabetes. Cytokine Growth Factor Rev 28: 71-77, 2016.

22. Fountoulakis N, Maltese G, Gnudi L and Karalliedde J: Reduced levels of anti-ageing hormone Klotho predict renal function decline in type 2 diabetes. J Clin Endocrinol Metab 103: 2026-2032, 2018

23. Zhou X and Wang X: Klotho: A novel biomarker for cancer. J Cancer Res Clin Oncol 141: 961-969, 2015.

24. Fakhar M, Najumuddin, Gul M and Rashid S: Antagonistic role of Klotho-derived peptides dynamics in the pancreatic cancer treatment through obstructing WNT-1 and Frizzled binding. Biophys Chem 240: 107-117, 2018.

25. Li Q, Li Y, Liang L, Li J, Luo D, Liu Q, Cai S and Li X: Klotho negatively regulated aerobic glycolysis in colorectal cancer via ERK/HIF1alpha axis. Cell Commun Signal 16: 26, 2018.

26. Pako J, Bikov A, Barta I, Matsueda H, Puskas R, Galffy G, Kerpel-Fronius A, Antus B and Horvath I: Assessment of the circulating klotho protein in lung cancer patients. Pathol Oncol Res 26: 233-238, 2020.

27. Wang N, Ma J, Ren Y, Xiang S and Jia R: Secreted klotho from exosomes alleviates inflammation and apoptosis in acute pancreatitis. Am J Transl Res 11: 3375-3383, 2019.

28. Sugiura H, Yoshida T, Mitobe M, Yoshida S, Shiohira S, Nitta K and Tsuchiya K: Klotho reduces apoptosis in experimental ischaemic acute kidney injury via HSP-70. Nephrol Dial Transplant 25: 60-68,2010.

29. Kim SJ, Cheresh P, Eren M, Jablonski RP, Yeldandi A, Ridge KM, Budinger GR, Kim DH, Wolf M, Vaughan DE, et al: Klotho, an antiaging molecule, attenuates oxidant-induced alveolar epithelial cell mtDNA damage and apoptosis. Am J Physiol Lung Cell Mol Physiol 313: L16-L26, 2017.

30. Zhu H, Gao Y, Zhu S, Cui Q and Du J: Klotho Improves cardiac function by suppressing reactive oxygen species (ROS) mediated apoptosis by modulating Mapks/Nrf2 signaling in doxorubicin-induced cardiotoxicity. Med Sci Monit 23: 5283-5293, 2017.

31. Song S, Gao P, Xiao H, Xu Y and Si LY: Klotho suppresses cardiomyocyte apoptosis in mice with stress-induced cardiac injury via downregulation of endoplasmic reticulum stress. PLoS One 8: e82968, 2013.

32. Liang X, Li B, Huang Q, Liu D and Ma H: Klotho prevents DEX-induced apoptosis in MC3T3-E1 osteoblasts through the NF- $\kappa$ B signaling pathway. Biochem Biophys Res Commun 507: 355-361, 2018.

33. Cui W, Leng B, Liu W and Wang G: Suppression of apoptosis in human umbilical vein endothelial cells (HUVECs) by Klotho protein is associated with reduced endoplasmic reticulum oxidative stress and activation of the PI3K/AKT pathway. Med Sci Monit 24: 8489-8499, 2018

34. Aijaz S, Ahmed N, Akhter Z, Sattar S, Lakhani S, Malik R and Pathan A: Clinical characteristics and in-hospital outcome in percutaneous coronary interventions with ST elevation myocardial infarction patients developing acute kidney injury. J Pak Med Assoc 69: 1827-1833, 2019.

35. Wang C, Pei YY, Ma YH, Ma XL, Liu ZW and Zhu JH: Risk factors for acute kidney injury in patients with acute myocardial infarction. Chin Med J (Engl) 132: 1660-1665, 2019.

36. Wang DX, Huang Z, Li QJ, Zhong GQ, He Y, Huang WQ, Cao XL, Tu RH and Meng JJ: Involvement of HSP90 in ischemic postconditioning-induced cardioprotection by inhibition of the complement system, JNK and inflammation. Acta Cir Bras 35 e202000105, 2020.

This work is licensed under a Creative Commons Attribution-NonCommercial-NoDerivatives 4.0 International (CC BY-NC-ND 4.0) License. 